\title{
Cardiac structure and function in elite Native Hawaiian and Pacific Islander Rugby Football League athletes: an exploratory study
}

\author{
Christopher Johnson $^{1} \cdot$ Lynsey Forsythe ${ }^{1} \cdot$ John Somauroo $^{1} \cdot$ Michael Papadakis $^{2} \cdot$ Keith George $^{1}$. \\ David Oxborough ${ }^{1}$
}

Received: 12 October 2017 / Accepted: 22 November 2017 / Published online: 30 November 2017

(c) The Author(s) 2017. This article is an open access publication

\begin{abstract}
The aim of this exploratory study was to define the Athletes Heart (AH) phenotype in Native Hawaiian \& Pacific Islander (NH\&PI) Rugby Football League (RFL) athletes. Specifically, (1) to describe conventional echocardiographic indices of left ventricle (LV) and right ventricle (RV) structure and function in NH\&PI RFL players and matched RFL Caucasian controls (CC) and (2) to demonstrate LV and RV mechanics in these populations. Ethnicity is a contributory factor to the phenotypical expression of the AH. There are no data describing the cardiac phenotype in NH\&PI athletes. Twenty-one male elite NH\&PI RFL athletes were evaluated using conventional echocardiography and myocardial speckle tracking, allowing the assessment of global longitudinal strain ( $\varepsilon$ ) and strain rate (SR); and basal, mid and global radial and circumferential $\varepsilon$ and SR. Basal and apical rotation and twist were also assessed. Results were compared with age-matched Caucasian counterparts $(C C ; n=21)$. LV mass $\left[42 \pm 9\right.$ versus $\left.37 \pm 4 \mathrm{~g} /\left(\mathrm{m}^{2.7}\right)\right]$, mean LV wall thickness (MWT: $9.5 \pm 0.7$ and $8.7 \pm 0.4 \mathrm{~mm}$ ), relative wall thickness (RWT: $0.35 \pm 0.04$ and $0.31 \pm 0.03)$ and RV wall thickness $(5 \pm 1$ and $4 \pm 1 \mathrm{~mm}$, all $\mathrm{p}<0.05$ ) were greater in NH\&PI compared with CC. LV and RV cavity dimensions and standard indices of LV and RV systolic and diastolic function were similar between groups. NH\&PI demonstrated reduced peak LV mid circumferential $\varepsilon$ and early diastolic SR, as well as reduced global radial $\varepsilon$. There was reduced basal rotation at 25-35\% systole, reduced apical rotation at 25-40\% and 60-100\% systole and reduced twist at 85-95\% systole in NH\&PI athletes. There were no differences between the two groups in RV wall mechanics. When compared to Caucasian controls, NH\&PI rugby players have a greater LV mass, MWT and RWT with concomitant reductions in circumferential and twist mechanics. This data acts to prompt further research in NH\&PI athletes.
\end{abstract}

Keywords Athletes heart $\cdot$ Left ventricle $\cdot$ Right ventricle $\cdot$ Ethnicity $\cdot$ Echocardiography $\cdot$ Strain imaging

\section{Introduction}

The 'Athletes Heart' (AH) describes cardiac structural and functional adaptations that occur in response to chronic exercise training [1]. Although there is debate related to the magnitude and timing of adaptation, it is apparent that all cardiac chambers become enlarged with concomitant changes in function [2]. This adaptation often creates a challenge for the clinician when the athlete undergoes pre-participation

David Oxborough

d.1.oxborough@ljmu.ac.uk

1 Research Institute for Sport and Exercise Sciences, Tom Reilly Building, Liverpool John Moores University, Liverpool L3 3AF, UK

2 Cardiology Clinical Academic Group, St Georges, University of London, London, UK screening as these physiological changes can mimic those seen in conditions that may predispose an athlete to sudden cardiac death (SCD) [2].

Echocardiography is regarded as an important tool in differential diagnosis within athletic populations. Novel techniques such as speckle tracking echocardiography facilitates the assessment of global and regional cardiac mechanics to determine strain $(\varepsilon)$, strain rate (SR) and twist data in the AH [3, 4]. The application of this technique aids the conventional assessment of cardiac function and has been employed in the differential diagnosis of physiological and pathological adaptation [4].

Current clinical criteria to distinguish $\mathrm{AH}$ from inherited cardiomyopathies are largely derived from Caucasian athletes [2]. More recently, studies have attempted to explore the impact of ethnicity upon the AH phenotype, with greater LV wall thicknesses observed in African and 
Afro-Caribbean athletes compared to Caucasian athletes [5-7]. These structural differences exacerbate the challenges in the differential diagnosis in diverse multi-ethnic sporting populations. In addition, there are limited data on right ventricular (RV) structure and function in different ethnic groups [8] with no data on the left or right heart of athletes from a Native Hawaiian and Pacific Island (NH\&PI) origin.

Rugby Football League (RFL) athletes provide an ideal model for assessment of the AH due to moderate dynamic and static components of the sport [9]. The recent occurrence of high profile events of SCD within the sport suggests the need to investigate this population. RFL athletes are ethnically diverse, specifically with a high number of participants from the Native Hawaiian and Pacific Island (NH\&PI) ethnic group.

The aim of this exploratory study is to investigate the AH phenotype in NH\&PI RFL athletes. Specifically, (1) to describe conventional echocardiographic indices of $\mathrm{LV}$ and RV structure and function in NH\&PI RFL players and matched RFL Caucasian controls (CC) and (2) to demonstrate $\mathrm{LV}$ and RV mechanics in these populations.

\section{Methods}

\section{Study design and population}

Twenty-one male RFL athletes of NH\&PI ethnicity (mean age $\pm S D, 27 \pm 3$ years) were recruited into this crosssectional study. Results were compared to a group of agematched male elite RFL athletes of Caucasian ethnicity (mean age $\pm \mathrm{SD}, 25 \pm 4$ years). All participants were free of known cardiovascular disease and avoided alcohol and caffeine consumption $24 \mathrm{~h}$ prior to data collection, whilst refraining from training for at least $6 \mathrm{~h}$ prior to the examinations. Ethics approval was granted by the Ethics Committee of Liverpool John Moores University.

\section{Procedures}

Blood pressure (BP), body mass (Seca 217, Hannover, Germany) and height (Seca Supra 719, Hannover, Germany) were recorded. Body surface area (BSA) was calculated as previously described [10]. All participants completed a health questionnaire to exclude cardiovascular symptoms, family history of sudden cardiac death and any other cardiovascular history and/or abnormalities. Ethnicity was self-reported by the athletes using the health questionnaire and subsequently recorded for future reference. A 12-lead ECG (CardioExpress SL6, Spacelab Healthcare, Washington, USA) was used to determine training and non-training related abnormalities [11].
A standard echocardiogram was undertaken by a single experienced sonographer using a commercially available ultrasound system (Vivid Q, GE Healthcare, Horten, Norway) and a 1.5-4 MHz phased array transducer. All images were acquired in accordance with the American Society of Echocardiography (ASE) guidelines [12]. Images were stored in a raw DICOM format and exported to an offline workstation (EchoPac version 7.0, GE Healthcare, Horten, Norway) for subsequent analysis. All data was analysed by a single experienced sonographer. A minimum of three cardiac cycles were averaged for all acquisitions.

\section{Conventional 2D echocardiography}

Standard measurements were made in accordance with ASE guidelines [12]. LV linear dimensions (LVIDd and LVIDs) allowed the calculation of LV mass using the ASE corrected equation. To provide a comprehensive assessment of LV wall thickness, eight measurements were made from a parasternal short axis orientation at basal and mid-levels from the anteroseptum, infero-septum, posterior wall and lateral wall [13]. Mean wall thickness (MWT) was calculated as an average of all eight segments. Relative wall thickness (RWT) was calculated using the formula [(RWT $=$ IVSWTd + PWTd $) /$ LVIDd], where IVSWTd denotes diastolic basal interventricular septal wall thickness and PWTd denotes diastolic basal posterior wall thickness. LV end diastolic volume (LVEDV), LV end systolic volume (LVESV), stroke volume (SV) and ejection fraction (EF) were calculated using a Simpson's biplane method. Pulsed-wave Tissue Doppler Imaging (TDI) assessed the septum and lateral wall for systolic $\left(\mathrm{S}^{\prime}\right)$, early $\left(\mathrm{E}^{\prime}\right)$ and late $\left(\mathrm{A}^{\prime}\right)$ diastolic velocities.

The RV outflow tract (RVOT) was measured at three locations $\left(\mathrm{RVOT}_{\text {plax }}, \mathrm{RVOT}_{1}\right.$ and $\left.\mathrm{RVOT}_{2}\right)$. The RV inflow was measured from a modified apical four chamber orientation and included the base $\left(\mathrm{RVD}_{1}\right)$, the mid-level $\left(\mathrm{RVD}_{2}\right)$ and the length $\left(\mathrm{RVD}_{3}\right)$. $\mathrm{RV}$ diastolic area (RVDa) and $\mathrm{RV}$ systolic area (RVSa) were measured and RV fractional area change was calculated (RVFAC). RV wall thickness (RVWT) was measured from a sub-costal approach. Tricuspid annular plane systolic excursion (TAPSE) was measured and TDI allowed for assessment of RV lateral $\mathrm{S}^{\prime}, \mathrm{E}^{\prime}$ and $\mathrm{A}^{\prime}$.

All structural indices were scaled allometrically to BSA based on the principle of geometrical similarity [14, 15]. Linear dimensions were scaled to $\mathrm{BSA}^{0.5}$, areas directly to BSA and volumes to BSA ${ }^{1.5}$, with LV mass also scaled to height $^{2.7}[16]$.

\section{Myocardial speckle tracking}

All images were acquired at a frame rate maintained between 40 and 90 frames per second, and settings were adjusted to provide optimal endocardial delineation. 
Images were analysed offline (EchoPac, Version 7.0, GE Healthcare, Horten, Norway). The ROI was adjusted to encompass the whole myocardium.

LV longitudinal $\varepsilon$ and SR were assessed using the apical four-chamber, two-chamber and three-chamber views, providing a global value based on the average of $18 \mathrm{seg}$ ments. The parasternal short-axis view allowed the assessment of LV basal circumferential and radial $\varepsilon$ and SR. Peak values were averaged from six myocardial segments assessed at the level of the mitral valve, whilst the level at the papillary muscle provided the same data at mid-level. The average global circumferential and radial $\varepsilon$ and SR was calculated from basal and mid values and hence 12 myocardial segments.

Basal rotation was measured using the circumference of the LV at the mitral valve level. A parasternal shortaxis view at the level of the apex, defined as the level just above the point of systolic cavity obliteration, was used to assess apical rotation. LV twist was calculated as the net difference between apical and basal rotation.

$\mathrm{RV}$ longitudinal $\varepsilon$ and SR were assessed using a modified four-chamber view. RV global longitudinal $\varepsilon$ and SR were averaged from three myocardial segments with the ROI restricted to the lateral wall only.

\section{Temporal analysis}

Raw data from STE assessment was exported into a spreadsheet and processed using cubic spline interpolation. Temporal data across systole and diastole in 5\% increments of absolute cardiac cycle were defined. Average $\varepsilon$ and SR values across each 5\% increment were used to create temporal curves and graphs.

\section{Statistical analysis}

Study data were collected and managed using REDCap electronic data capture tools hosted at Liverpool John Moores University [17]. All echocardiographic data are presented as mean \pm SD. Statistical analyses were performed using SPSS Version 23.0 for Windows (SPSS, Chicago, Illinois, USA). All variables, including $\varepsilon$ and SR at each 5\% increment time point, were analysed for normality using a Shapiro-Wilk test. All normally distributed variables were analysed between groups using an independent $\mathrm{T}$ test, with non-normally distributed variables analysed using a non-parametric Mann-Whitney U test. $\varepsilon$ and SR $5 \%$ increments for systole and diastole were analysed for group by time interactions using a Two-Way ANOVA. The $\mathrm{p}$ value of $<0.05$ was considered statistically significant.
Table 1 Echocardiographic parameters of the LV and RV

\begin{tabular}{|c|c|c|c|}
\hline \multirow[t]{2}{*}{ Parameter } & \multicolumn{2}{|l|}{ Mean \pm SD } & \multirow[t]{2}{*}{$\mathrm{p}$ value } \\
\hline & NH\&PI & $\mathrm{CC}$ & \\
\hline \multicolumn{4}{|l|}{ Left ventricle } \\
\hline IVSWTd (mm) & $10 \pm 1$ & $9 \pm 1$ & $0.006^{*}$ \\
\hline PWTd (mm) & $10 \pm 1$ & $9 \pm 1$ & $0.012 *$ \\
\hline $\operatorname{LVIDd}\left[\mathrm{mm} /\left(\mathrm{m}^{2}\right)^{0.5}\right]$ & $37 \pm 3$ & $38 \pm 2$ & 0.229 \\
\hline LVIDs $\left[\mathrm{mm} /\left(\mathrm{m}^{2}\right)^{0.5}\right]$ & $26 \pm 2$ & $26 \pm 2$ & 0.878 \\
\hline $\mathrm{LV}$ mass $\left[\mathrm{g} /\left(\mathrm{m}^{2}\right)^{2.7}\right]$ & $42 \pm 9$ & $37 \pm 4$ & $0.017 *$ \\
\hline MWT (mm) & $10 \pm 0.7$ & $9 \pm 0.4$ & $<0.001 *$ \\
\hline RWT & $0.35 \pm 0.04$ & $0.31 \pm 0.03$ & $0.003 *$ \\
\hline $\operatorname{LVEDV}\left[\mathrm{ml} /\left(\mathrm{m}^{2}\right)^{1.5}\right]$ & $47 \pm 8$ & $47 \pm 7$ & 0.873 \\
\hline $\operatorname{LVESV}\left[\mathrm{ml} /\left(\mathrm{m}^{2}\right)^{1.5}\right]$ & $19 \pm 4$ & $20 \pm 4$ & 0.566 \\
\hline LV SV (ml) & $94 \pm 19$ & $89 \pm 13$ & 0.27 \\
\hline LV EF (\%) & $59 \pm 4$ & $58 \pm 5$ & 0.515 \\
\hline Septal S' $(\mathrm{cm} / \mathrm{s})$ & $9 \pm 1.3$ & $9.3 \pm 1.1$ & 0.378 \\
\hline Septal E' $(\mathrm{cm} / \mathrm{s})$ & $11.7 \pm 1.5$ & $12.5 \pm 2.4$ & 0.191 \\
\hline Septal A' $(\mathrm{cm} / \mathrm{s})$ & $7.5 \pm 1.6$ & $7.8 \pm 1.6$ & 0.575 \\
\hline Lateral $\mathrm{S}^{\prime}(\mathrm{cm} / \mathrm{s})$ & $11.5 \pm 2.9$ & $11.6 \pm 2.4$ & 0.861 \\
\hline Lateral $\mathrm{E}^{\prime}(\mathrm{cm} / \mathrm{s})$ & $17 \pm 3.4$ & $18.5 \pm 2.7$ & 0.108 \\
\hline Lateral A' $(\mathrm{cm} / \mathrm{s})$ & $7.7 \pm 2.7$ & $6.6 \pm 2$ & 0.120 \\
\hline \multicolumn{4}{|l|}{ Right ventricle } \\
\hline $\mathrm{RVOT}_{\text {PLAX }}$ index $\left[\mathrm{mm} /\left(\mathrm{m}^{2}\right)^{0.5}\right]$ & $23 \pm 2$ & $23 \pm 2$ & 0.981 \\
\hline $\mathrm{RVOT}_{1}$ index $\left[\mathrm{mm} /\left(\mathrm{m}^{2}\right)^{0.5}\right]$ & $23 \pm 3$ & $24 \pm 3$ & 0.476 \\
\hline $\mathrm{RVOT}_{2}$ index $\left[\mathrm{mm} /\left(\mathrm{m}^{2}\right)^{0.5}\right]$ & $18 \pm 2$ & $19 \pm 3$ & 0.212 \\
\hline $\mathrm{RVD}_{1}$ index $\left[\mathrm{mm} /\left(\mathrm{m}^{2}\right)^{0.5}\right]$ & $29 \pm 2$ & $30 \pm 4$ & 0.229 \\
\hline $\mathrm{RVD}_{2}$ index $\left[\mathrm{mm} /\left(\mathrm{m}^{2}\right)^{0.5}\right]$ & $23 \pm 3$ & $21 \pm 3$ & 0.085 \\
\hline $\mathrm{RVD}_{3}$ index $\left[\mathrm{mm} /\left(\mathrm{m}^{2}\right)^{0.5}\right]$ & $56 \pm 13$ & $62 \pm 6$ & 0.054 \\
\hline RVDa Index $\left(\mathrm{cm} / \mathrm{m}^{2}\right)$ & $13 \pm 2$ & $13 \pm 2$ & 0.399 \\
\hline RVSa Index $\left(\mathrm{cm} / \mathrm{m}^{2}\right)$ & $7 \pm 1$ & $7 \pm 2$ & 0.809 \\
\hline RVWT (mm) & $5 \pm 1$ & $4 \pm 1$ & $0.016 *$ \\
\hline TAPSE (mm) & $24 \pm 4$ & $25 \pm 4$ & 0.742 \\
\hline RVFAC (\%) & $47 \pm 6$ & $49 \pm 7.2$ & 0.365 \\
\hline $\mathrm{RV} \mathrm{S}^{\prime}(\mathrm{cm} / \mathrm{s})$ & $15 \pm 2$ & $14 \pm 2$ & 0.442 \\
\hline $\mathrm{RV} \mathrm{E}^{\prime}(\mathrm{cm} / \mathrm{s})$ & $14 \pm 3$ & $15 \pm 3$ & 0.27 \\
\hline $\mathrm{RV} \mathrm{A}^{\prime}(\mathrm{cm} / \mathrm{s})$ & $11 \pm 2$ & $10 \pm 3$ & 0.161 \\
\hline
\end{tabular}

$L V$ left ventricle, $R V$ right ventricle, $N H \& P I$ Native Hawaiian \& Pacific Islanders, $C C$ Caucasian Controls, $d$ at end-diastole, $s$ at endsystole, IVSWT interventricular septal wall thickness, $P W T$ posterior wall thickness, $L V I D L V$ internal diameter, $M W T$ mean wall thickness, $R W T$ relative wall thickness, $L V E D V L V$ end-diastolic volume, $L V E S V L V$ end-systolic volume, $S V$ stroke volume, $E F$ ejection fraction, RVOT RV outflow tract, $R V D \mathrm{RV}$ dimension, $R V D a \mathrm{RV}$ area at end-diastole, $R V S a \mathrm{RV}$ area at end-systole, $R V W T \mathrm{RV}$ wall thickness, TAPSE tricuspid annular plane systolic excursion, RVFAC RV fractional area change

$* \mathrm{p}<0.05 \mathrm{NH} \& \mathrm{PI}$ versus CC

\section{Results}

Both the NH\&PI and CC were similar $(\mathrm{p}>0.05)$ in height $(1.83 \pm 0.06$ and $1.83 \pm 0.05 \mathrm{~m})$, BSA $(2.29 \pm 0.16$ and $\left.2.21 \pm 0.12 \mathrm{~kg} / \mathrm{m}^{2}\right)$, heart rate $(57 \pm 12$ and $55 \pm 9 \mathrm{bpm})$, 
cardiac output $(5.26 \pm 1.09$ and $4.8 \pm 0.66 \mathrm{~L} / \mathrm{min})$, systolic $\mathrm{BP}(131 \pm 10$ and $129 \pm 8 \mathrm{mmHg})$, diastolic BP $(70 \pm 7$ and $69 \pm 9 \mathrm{mmHg})$, and training hours $(23 \pm 10$ and $23 \pm 7 \mathrm{~h} /$ week). NH\&PI athletes had a significantly larger body mass than the CC $(103 \pm 11$ and $96 \pm 8 \mathrm{~kg})$. There were no differences between groups for ECG criteria. Normal ECG findings included sinus bradycardia (NH\&PI 57\%, CC 76\%), sinus arrhythmia (NH\&PI 10\%, CC 10\%), 1 st degree AV block (NH\&PI 19\% and CC 5\%), partial RBBB (NH\&PI 14\%, CC 10\%), early repolarisation (NH\&PI 95\%, CC 67\%) and isolated criteria for LVH (NH\&PI 14\%, CC 19\%) [11]. T wave inversion $>2 \mathrm{~mm}$ in the anterior leads was found in one athlete from each ethnic group. On subsequent analysis, these findings were considered physiological in both cases as determined by echocardiography and cardiac magnetic resonance imaging.

\section{Conventional structure and function}

Structural and standard functional indices of the LV and RV are presented in Table 1 and Fig. 1. LV mass, MWT, RWT and RVWT were significantly larger in NH\&PI compared with CC. There were no between-group differences in LV and RV cavity dimensions and all standard systolic and diastolic functional indices.

\section{Mechanics}

\section{Peak data}

Peak LV and RV $\varepsilon$ and SR data are presented in Table 2. LV mid circumferential peak $\varepsilon, \mathrm{LV}$ mid circumferential SRE, LV global circumferential SRE and LV global radial SRE were significantly lower in the NH\&PI compared to the CC. All other peak LV mechanical data, including twist indices and peak RV mechanical data, were similar between groups.

\section{Temporal analysis}

Significant group by time interactions $(\mathrm{p}<0.05)$ were present for mid circumferential strain in systole, with systolic twist producing a trend towards significance $(p=0.058)$. There were also significant interactions during diastole for global and mid circumferential, and mid radial SR, as well as apical twist.

Individual systolic and diastolic 5\% increment analysis demonstrated global circumferential $\varepsilon$ (late diastole), mid circumferential $\varepsilon$ (late systole through to early diastole and late diastole) and mid radial $\varepsilon$ (early systole) as lower in NH\&PI athletes (see Fig. 2). Likewise, there was evidence of reduced SR in NH\&PI athletes (global longitudinal SR in early diastole; global, mid and basal circumferential SR in early diastole; global and mid radial SR in early diastole; global circumferential SR in late diastole). There was also evidence of reduced basal rotation at $25-35 \%$ systole, reduced apical rotation at $25-40 \%$ and $60-100 \%$ systole and reduced twist at $85-95 \%$ systole in NH\&PI athletes (see Fig. 3). On the contrary, there were no temporal differences in any RV mechanical data (see Fig. 2).

\section{Discussion}

The main findings of this exploratory study are (1) NH\&PI athletes have larger LVM, MWT, RWT and RVWT compared to CC with no between-group differences in standard $\mathrm{LV}$ and RV conventional functional indices and (2) there is evidence of reduced peak and temporal LV mechanics but no difference in RV mechanics in NH\&PI athletes compared to $\mathrm{CC}$.

\section{Structural adaptation}

In the current study the NH\&PI demonstrated larger LV mass and thicker LV walls than $\mathrm{CC}$ with no significant
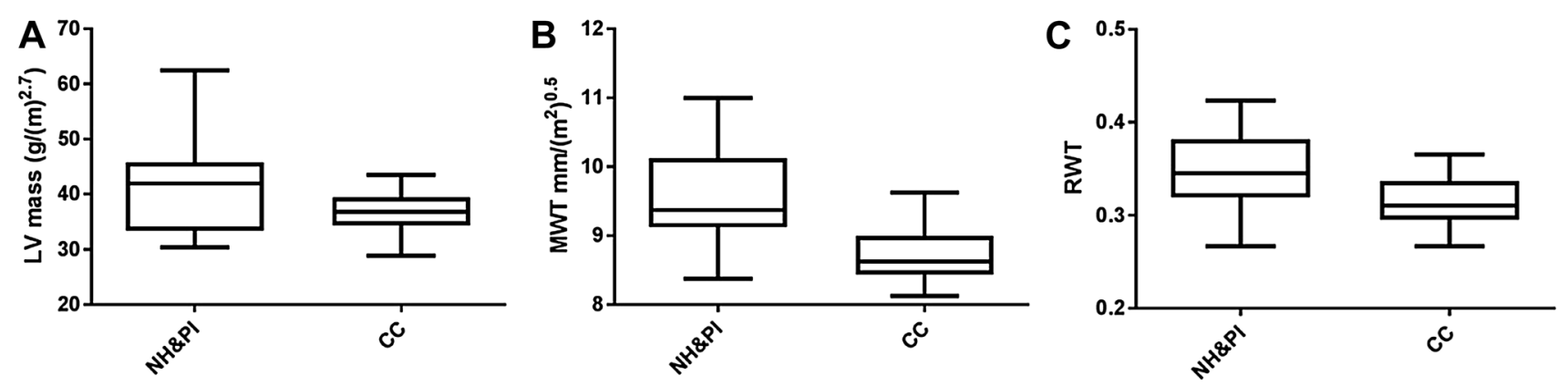

Fig. 1 Box plot demonstrating, a LV mass, b mean wall thickness, $\mathbf{c}$ relative wall thickness between NH\&PI and CC 
differences in LV cavity dimensions. The combination of larger LV mass, thicker LV walls and similar LV cavity dimensions in NH\&PI meant that although none of the athletes met the absolute criteria for concentric remodelling, they had values closer to the cut-off [12]. A similar pattern of increased wall thicknesses in African/Afro-Caribbean athletes compared to other ethnic backgrounds has previously been demonstrated [5, 6]. Although previous studies have described resting BP differences between ethnicities [18], our study reports similar resting BP between the NH\&PI and CC. Therefore, the known notion that haemodynamic stress in the form of an increased BP increases ventricular afterload causing adaptation in ventricular wall thickness does not explain the current differences found between the NH\&PI and CC. Therefore, we suggest a possible difference in the response of the LV to alterations in cardiac load during exercise training in NH\&PI athletes, when compared to a CC population, which could follow a similar pattern to their African counterparts [5, 6]. It is important to note that currently there is no data pertaining to the acute cardiac responses to exercise in the NH\&PI population, and we believe this should be further investigated. This phenomenon has also been observed in a pathological response to pressure overload in hypertensive disease [19] and therefore further work should aim to establish any possible implications to the NH\&PI population.

RV structure in NH\&PI athletes followed a similar pattern to the phenotype seen in the LV with increased RVWT but similar RV cavity dimensions. Zaidi et al. [8] investigated the RV in athletes of African and Afro-Caribbean origin compared to a CC athletic population. RVOT values were smaller in the African athletic population compared to CC but with a trend towards larger RVWT $(p=0.05)$. Our findings in NH\&PI suggest that these athletes have a similar adaptive response to the exercise stimulus in the RV which may be explained through evidence of a concomitant/balanced increase in $\mathrm{RV}$ and $\mathrm{LV}$ adaptations in respect to mass and volume [20].

This type of AH morphology may exacerbate the difficulty in distinguishing physiology from pathology in NH\&PI athletes [21]. That aside, it is important to note that none of the athletes in this study had absolute LV wall thicknesses greater than $12 \mathrm{~mm}$. However, RFL is a moderate static and dynamic sport and it is speculative to suggest that NH\&PI athletes engaged in other sports [9] may present with even greater wall thicknesses compared to their Caucasian counterparts [22].

\section{Functional adaptation}

Although systolic function as determined by conventional imaging was similar between ethnic groups, reduced circumferential and radial mechanics in systole and diastole
Table $2 \mathrm{LV}$ and $\mathrm{RV} \varepsilon$ and SR

\begin{tabular}{|c|c|c|c|}
\hline \multirow[t]{2}{*}{ Parameter } & \multicolumn{2}{|l|}{ Mean \pm SD } & \multirow[t]{2}{*}{$\mathrm{p}$ value } \\
\hline & NH\&PI & $\mathrm{CC}$ & \\
\hline \multicolumn{4}{|l|}{ LV longitudinal } \\
\hline$\varepsilon(\%)$ & $-19 \pm 2$ & $-19 \pm 2$ & 0.897 \\
\hline Time to peak $\varepsilon$ (s) & $0.38 \pm 0.03$ & $0.37 \pm 0.03$ & 0.325 \\
\hline $\operatorname{SRS}\left(S^{-1}\right)$ & $-0.93 \pm 0.09$ & $-0.95 \pm 0.1$ & 0.549 \\
\hline $\operatorname{SRE}\left(S^{-1}\right)$ & $1.29 \pm 0.18$ & $1.36 \pm 0.18$ & 0.229 \\
\hline SRA $\left(S^{-1}\right)$ & $0.67 \pm 0.16$ & $0.62 \pm 0.14$ & 0.294 \\
\hline \multicolumn{4}{|l|}{ LV circumferential } \\
\hline$\varepsilon(\%)$ & $-20 \pm 3$ & $-21 \pm 2$ & 0.094 \\
\hline Time to peak $\varepsilon(\mathrm{s})$ & $0.38 \pm 0.03$ & $0.37 \pm 0.03$ & 0.785 \\
\hline $\operatorname{SRS}\left(\mathrm{S}^{-1}\right)$ & $-1.09 \pm 0.2$ & $-1.17 \pm 0.12$ & 0.154 \\
\hline $\operatorname{SRE}\left(S^{-1}\right)$ & $1.52 \pm 0.35$ & $1.8 \pm 0.37$ & $0.023^{*}$ \\
\hline SRA $\left(S^{-1}\right)$ & $0.43 \pm 0.13$ & $0.45 \pm 0.09$ & 0.659 \\
\hline$\varepsilon \operatorname{mid}(\%)$ & $-20 \pm 3$ & $-21 \pm 2$ & $0.029 *$ \\
\hline $\operatorname{Mid} \operatorname{SRS}\left(\mathrm{S}^{-1}\right)$ & $-1.11 \pm 0.21$ & $-1.18 \pm 0.16$ & 0.261 \\
\hline $\operatorname{Mid} \operatorname{SRE}\left(\mathrm{S}^{-1}\right)$ & $1.44 \pm 0.34$ & $1.7 \pm 0.38$ & $0.019^{*}$ \\
\hline $\operatorname{Mid}$ SRA $\left(\mathrm{S}^{-1}\right)$ & $0.51 \pm 0.12$ & $0.5 \pm 0.14$ & 0.973 \\
\hline$\varepsilon$ basal $(\%)$ & $-20 \pm 4$ & $-21 \pm 3$ & 0.347 \\
\hline Basal SRS $\left(\mathrm{S}^{-1}\right)$ & $-1.14 \pm 0.2$ & $-1.21 \pm 0.14$ & 0.222 \\
\hline Basal SRE $\left(\mathrm{S}^{-1}\right)$ & $1.69 \pm 0.51$ & $1.91 \pm 0.45$ & 0.153 \\
\hline Basal SRA $\left(\mathrm{S}^{-1}\right)$ & $0.4 \pm 0.21$ & $0.39 \pm 0.09$ & 0.703 \\
\hline \multicolumn{4}{|l|}{ LV radial } \\
\hline$\varepsilon(\%)$ & $43 \pm 14$ & $50 \pm 12$ & 0.112 \\
\hline Time to peak $\varepsilon$ (s) & $0.41 \pm 0.04$ & $0.40 \pm 0.5$ & 0.697 \\
\hline $\operatorname{SRS}\left(S^{-1}\right)$ & $1.57 \pm 0.31$ & $1.66 \pm 0.34$ & 0.371 \\
\hline $\operatorname{SRE}\left(S^{-1}\right)$ & $-1.79 \pm 0.34$ & $-2.1 \pm 0.43$ & $0.013^{*}$ \\
\hline SRA $\left(S^{-1}\right)$ & $-1.01 \pm 0.56$ & $-0.94 \pm 0.33$ & 0.87 \\
\hline$\varepsilon \operatorname{mid}(\%)$ & $43 \pm 18$ & $51 \pm 16$ & 0.13 \\
\hline $\operatorname{Mid} \operatorname{SRS}\left(\mathrm{S}^{-1}\right)$ & $1.71 \pm 0.47$ & $1.68 \pm 0.37$ & 0.825 \\
\hline $\operatorname{Mid} \operatorname{SRE}\left(\mathrm{S}^{-1}\right)$ & $-1.99 \pm 0.57$ & $-2.33 \pm 0.81$ & 0.122 \\
\hline $\operatorname{Mid} \operatorname{SRA}\left(\mathrm{S}^{-1}\right)$ & $-1.12 \pm 0.66$ & $-0.96 \pm 0.45$ & 0.367 \\
\hline$\varepsilon$ basal $(\%)$ & $43 \pm 4$ & $58 \pm 3$ & 0.195 \\
\hline Basal SRS $\left(\mathrm{S}^{-1}\right)$ & $1.81 \pm 0.46$ & $1.93 \pm 0.55$ & 0.434 \\
\hline Basal SRE $\left(\mathrm{S}^{-1}\right)$ & $-1.6 \pm 0.53$ & $-1.87 \pm 0.53$ & 0.099 \\
\hline Basal SRA $\left(\mathrm{S}^{-1}\right)$ & $-0.91 \pm 0.57$ & $-0.93 \pm 0.4$ & 0.917 \\
\hline \multicolumn{4}{|l|}{ LV rotation } \\
\hline Apical rotation $\left(^{\circ}\right)$ & $7.1 \pm 3.8$ & $9.3 \pm 3.6$ & 0.054 \\
\hline Basal rotation $\left({ }^{\circ}\right)$ & $-6.9 \pm 2.6$ & $-6.7 \pm 3.5$ & 0.873 \\
\hline Twist $\left({ }^{\circ}\right)$ & $13.4 \pm 4.6$ & $15.6 \pm 5.1$ & 0.159 \\
\hline \multicolumn{4}{|l|}{ RV longitudinal } \\
\hline$\varepsilon(\%)$ & $-27 \pm 3$ & $-27 \pm 4$ & 0.857 \\
\hline Time to peak $\varepsilon$ (s) & $0.39 \pm 0.03$ & $0.38 \pm 0.03$ & 0.252 \\
\hline $\operatorname{SRS}\left(\mathrm{S}^{-1}\right)$ & $-1.24 \pm 0.2$ & $-1.31 \pm 0.21$ & 0.266 \\
\hline $\operatorname{SRE}\left(\mathrm{S}^{-1}\right)$ & $1.60 \pm 0.35$ & $1.56 \pm 0.36$ & 0.771 \\
\hline SRA $\left(S^{-1}\right)$ & $0.82 \pm 0.26$ & $0.84 \pm 0.28$ & 0.642 \\
\hline
\end{tabular}

$L V$ left ventricle, $R V$ right ventricle, $\varepsilon$ strain, $S R$ strain rate, $N H \& P I$ Native Hawaiian \& Pacific Islanders, $C C$ caucasian controls $* \mathrm{p}<0.05 \mathrm{NH} \& \mathrm{PI}$ versus CC 

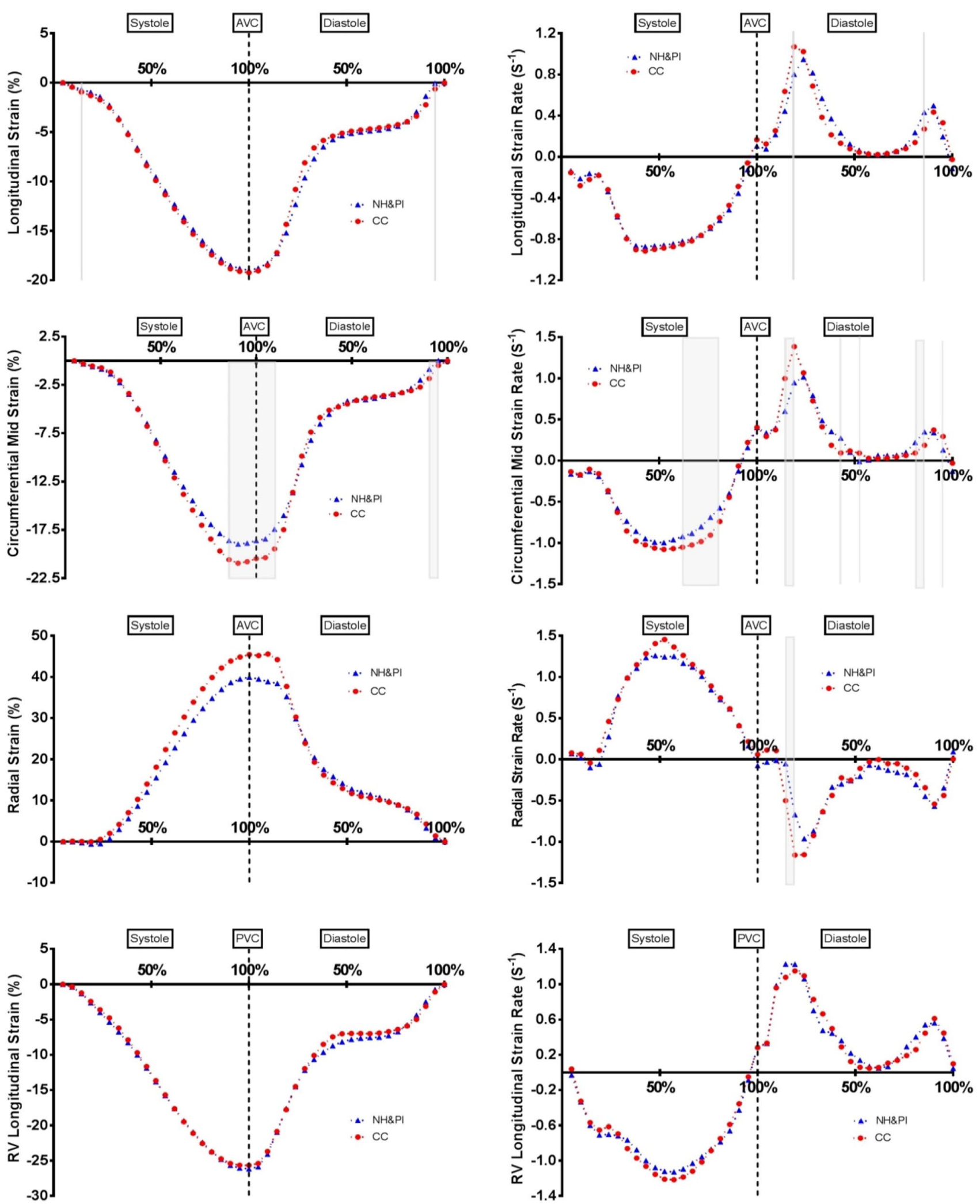

Fig. 2 Left ventricular longitudinal, mid circumferential, radial and right ventricular longitudinal $\varepsilon$ and strain rate-grey area denotes statistical significance $(\mathrm{p}<0.05)$. AVC Aortic valve closure, $P V C$ pulmonary valve closure 

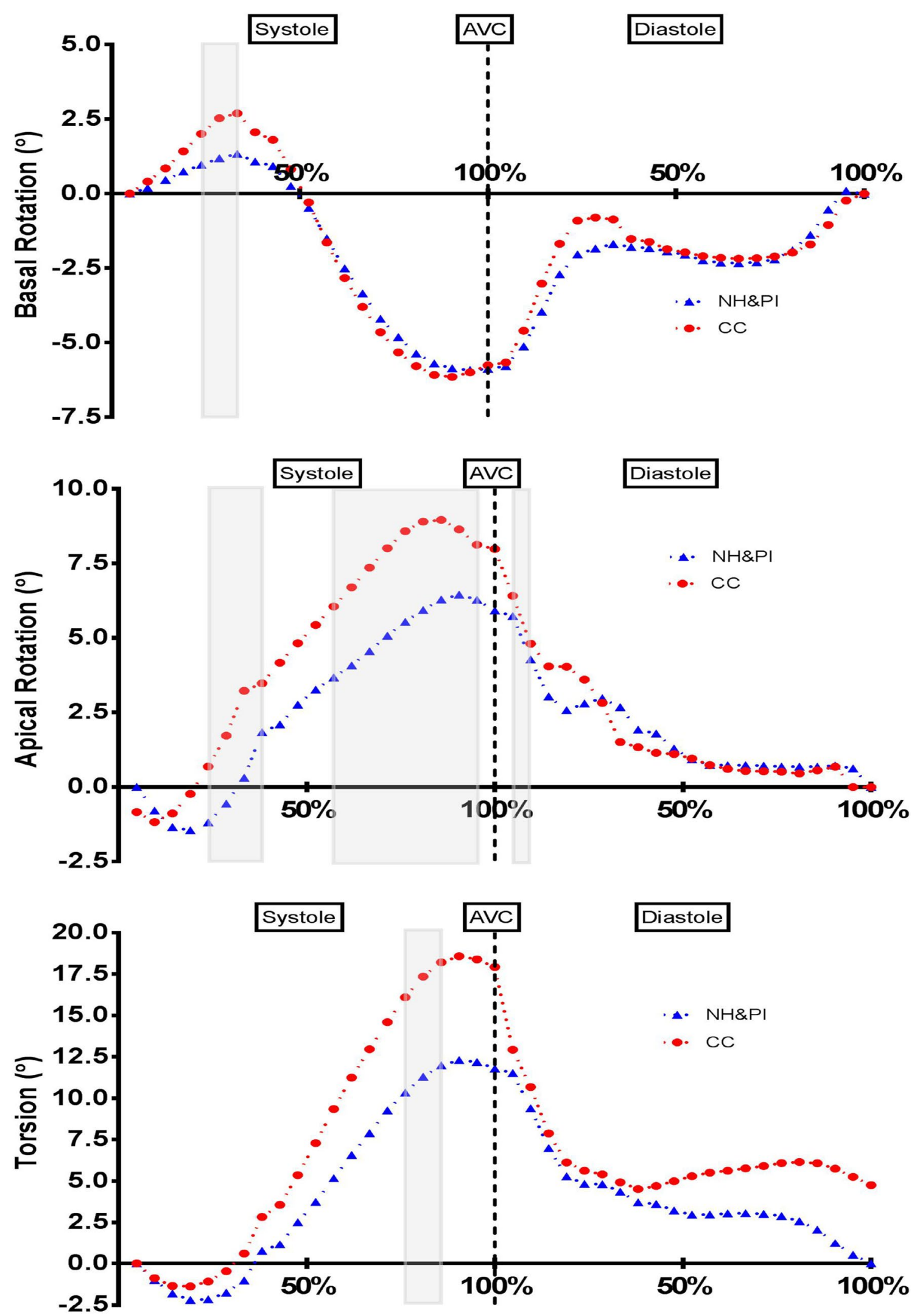

Fig. 3 Left ventricular basal rotation, apical rotation and torsion — grey area denotes statistical significance $(\mathrm{p}<0.05)$ 
were observed in NH\&PI athletes. It is difficult to directly attribute these findings to the differences in LV geometry but reduced peak circumferential $\varepsilon$ has previously been reported in body builders who exhibited increased LV mass and wall thickness compared to a marathon runner cohort [23]. It may be that adequate LV forces are generated with lower circumferential contribution. The McIver Townsend hypothesis [24] may partially explain this phenomenon. The hypothesis states that alterations in mechanical function are a product of an increase in LV hypertrophy (LVH). Essentially, radial displacement is maintained even in the presence of reduced longitudinal function and non-compensatory circumferential function. $\mathrm{LVH}$, and therefore myocardial muscle volume, leads to an increased endocardial displacement for any given level of deformation. These findings need to be reproduced in a much larger NH\&PI population but these exploratory findings are important from both a physiological and clinical perspective.

In addition to circumferential mechanics we also observed significantly reduced apical and basal rotation in early systole consistent with an extended pre-twist period in the NH\&PI. A reduction in apical rotation throughout systole resulted in lower twist mechanics. It could be suggested that the alteration in early diastolic SR in the NH\&PI could be a consequence of the reduced apical rotation observed. Apical twist is considered an important component of LV function to store additional potential energy that is released to increase early diastolic suction [25]. This "recoil" causes a rapid reduction of $\mathrm{LV}$ pressure leading to early diastolic filling [25]. A reduced store of potential energy could attenuate the "recoil" relaxation of the ventricle. A previous deformation study [26] also highlighted that apical rotation is the primary determinant of peak systolic LV twist. It is not possible from the current study to conclude as to whether reduced apical rotation is due to a reduced mechanical functionality that could be linked to the increased wall thickness present, as seen in patients with HCM [27] or if it is an amplification of the previously identified adaptive response to training concluded as a "reserve" for the onset of exercise [28]. Therefore, as mentioned previously, further research is needed to evaluate the acute cardiac response to exercise within the NH\&PI population.

Our findings of similar RV mechanics in the presence of increased RVWT in NH\&PI athletes is reassuring and highlights a similar response to the LV in this ethnic group. It is important to note that it is not possible to accurately assess RV circumferential function and therefore a comprehensive RV functional assessment may provide further insight.

\section{Limitations}

This study is limited by a small sample size and hence we deemed this an exploratory study. However, PostHoc analysis of the MWT demonstrated $100 \%$ statistical power, therefore demonstrating adequate statistical power with this sample size.

It is also important to note that we selected our athletes for their sporting discipline, however, these findings cannot be generalizable to athletes of other sports, sex or age. Hence further work to address the multi-factorial nature of the $\mathrm{AH}$ alongside ethnic specific adaptation is required.

It is important to note that longitudinal $\varepsilon$ is the most commonly used indicator of LV mechanics in practice, with radial and circumferential $\varepsilon$ deemed to carry limitations in terms of reproducibility in both physiological and pathological subjects.

\section{Conclusion}

This study demonstrated that athletes of NH\&PI ethnicity have greater LV and RV wall thickness and mass compared to their Caucasian counterparts. In addition, there is reduced LV circumferential and twist mechanics that may reflect the differences in LV geometry structural adaptation.

\section{Compliance with ethical standards}

Conflict of interest The authors declare that they have no conflict of interest.

Ethical approval All procedures performed in studies involving human participants were in accordance with the ethical standards of the institutional and/or national research committee and with the 1964 Helsinki declaration and its later amendments or comparable ethical standards.

Informed consent Informed consent was obtained from all individual participants included in the study.

Open Access This article is distributed under the terms of the Creative Commons Attribution 4.0 International License (http://creativecommons.org/licenses/by/4.0/), which permits unrestricted use, distribution, and reproduction in any medium, provided you give appropriate credit to the original author(s) and the source, provide a link to the Creative Commons license, and indicate if changes were made.

\section{Appendix}

See Fig. 4. 

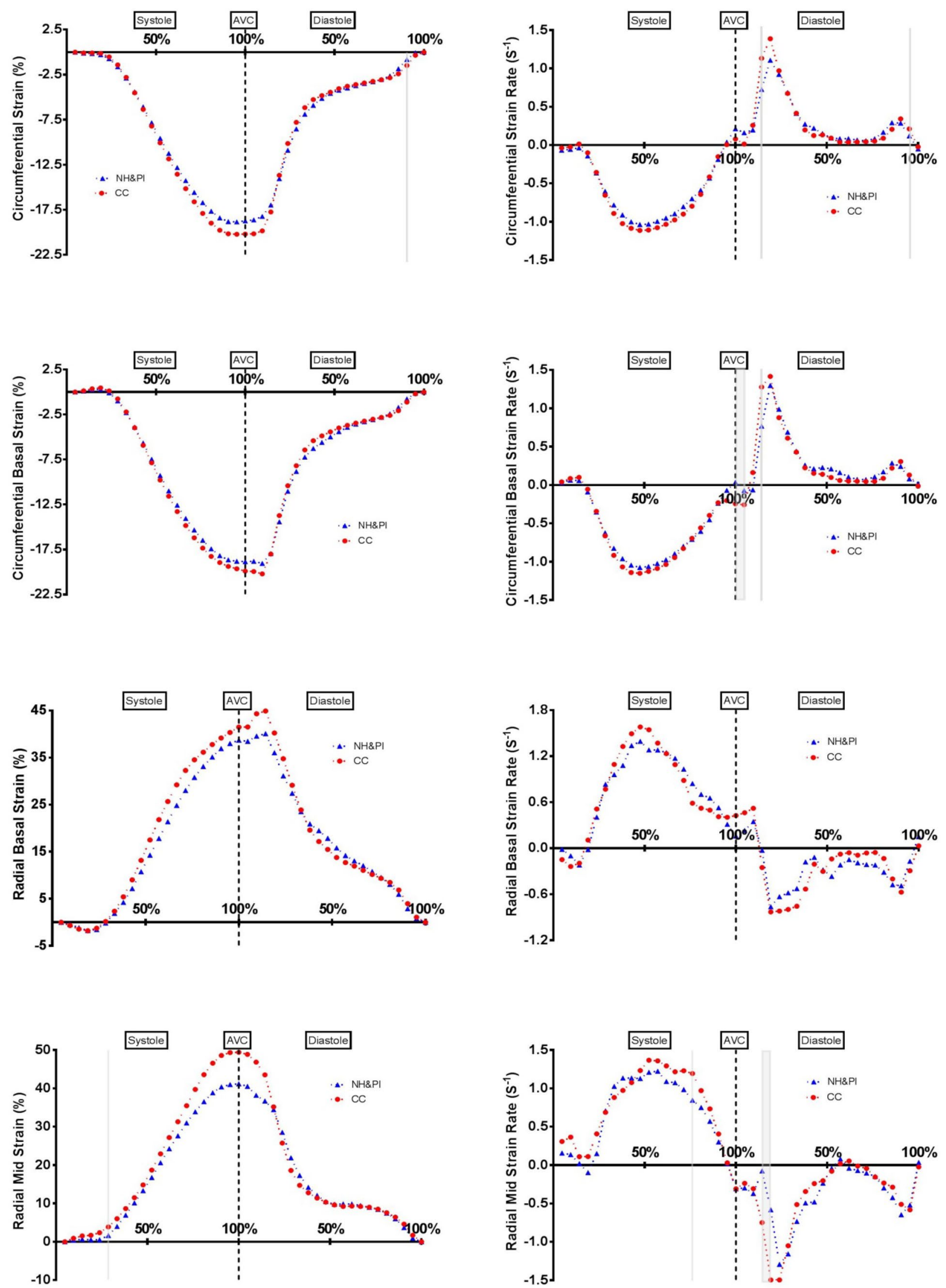

Fig. 4 Circumferential and radial mechanics 


\section{References}

1. Pluim BM, Zwinderman AH, Van Der Laarse A, Van Der Wall EE (2000) The athlete's heart: a meta-analysis of cardiac structure and function. Circulation 101:336-344

2. Prior DL, La Gerche A (2012) The athlete's heart. Heart 98: 947 $-55$

3. D'Ascenzi F, Caselli S, Solari M, Pelliccia A, Cameli M, Focardi $\mathrm{M}$ et al (2016) Novel echocardiographic techniques for the evaluation of athletes' heart: a focus on speckle-tracking echocardiography. Eur J Prev Cardiol 23:437-446

4. Utomi V, Oxborough D, Ashley E, Lord R, Fletcher S, Stembridge $M$ et al (2014) Predominance of normal left ventricular geometry in the male 'athlete's heart'. Heart 100:1264-1271

5. Basavarajaiah S, Boraita A, Whyte G, Wilson M, Carby L, Shah A et al (2008) Ethnic differences in left ventricular remodelling in highly-trained athletes relevance to differentiating physiologic left ventricular hypertrophy from hypertrophic cardiomyopathy. J Am Coll Cardiol 51:2256-2262

6. Papadakis M, Carre F, Kervio G, Rawlins J, Panoulas VF, Chandra $\mathrm{N}$ et al (2011) The prevalence, distribution, and clinical outcomes of electrocardiographic repolarization patterns in male athletes of African/Afro-Caribbean origin. Eur Heart J 32:2304-2313

7. Wilson MG, Chatard JC, Carre F, Hamilton B, Whyte GP, Sharma $S$ et al (2012) Prevalence of electrocardiographic abnormalities in West-Asian and African male athletes. Br J Sports Med 46:341-347

8. Zaidi A, Ghani S, Sharma R, Oxborough D, Panoulas VF, Sheikh $\mathrm{N}$ et al (2013) Physiological right ventricular adaptation in elite athletes of African and Afro-Caribbean origin. Circulation 127:1783-1792

9. Mitchell JH, Haskell W, Snell P, Van Camp SP (2005) Task force 8: classification of sports. J Am Coll Cardiol 45:1364-1367

10. Mosteller R (1987) Simplified calculation of body surface area. N Engl J Med 317:1098

11. Sharma S, Drezner J, Baggish A, Papadakis M, Wilson MG, Prutkin JM et al (2017) International recommendations for electrocardiographic interpretation in athletes. J Am Coll Cardiol 69:1057-1075

12. Lang RM, Badano LP, Mor-Avi V, Afilalo J, Armstrong A, Ernande L et al (2015) Recommendations for cardiac chamber quantification by echocardiography in adults: an update from the American Society of Echocardiography and the European Association of Cardiovascular Imaging. J Am Soc Echocardiogr 28:1-39

13. Wigle ED, Sasson Z, Henderson M, Ruddy TD, Fulop J, Rakowski $\mathrm{H}$ et al (1985) Hypertrophic cardiomyopathy. The importance of the site and the extent of hypertrophy. A review. Prog Cardiovasc Dis 28:1-83

14. Batterham A, George K (1998) Modeling the influence of body size and composition on M-mode echocardiographic dimensions. Am J Physiol Heart Circ Physiol 274:701-708
15. Dewey FE, Rosenthal D, Murphy DJ, Froelicher VF, Ashyley EA (2008) Does size matter? Clinical applications of scaling cardiac size and function for body size. Circulation 117:2279-2287

16. Daniels SR, Kimball TR, Morrison JA, Khoury P, Meyer RA (1995) Indexing left ventricular mass to account for differences in body size in children and adolescents without cardiovascular disease. Am J Cardiol 76:699-701

17. Harris PA, Taylor R, Thielke R, Payne J, Goonzalez N, Conde JG (2009) Research electronic data capture (REDCap) — a metadatadriven methodology and workflow process for providing translational research informatics support. J Biomed Inform 42:377-381

18. Lane D, Beevers DG, Lip GY (2002) Ethnic differences in blood pressure and the prevelance of hypertension in England. J Hum Hypertens 16:267-273

19. Kizer JR, Arnett DK, Bella JN, Paranicas M, Rao DC, Province MA et al (2004) Differences in left ventricular structure between black and white hypertensive adults. Hypertension 43:1182-1188

20. Scharhag J, Schneider G, Urhausen A, Rochette V, Kramann B, Kindermann W (2002) Athlete's heart: right and left ventricular mass and function in male endurance athletes and untrained individuals determined by magnetic resonance imaging. J Am Coll Cardiol 40:1856-1863

21. Maron BJ (2005) Distinguishing hypertrophic cardiomyopathy from athlete's heart: a clinical problem of increasing magnitude and significance. Heart 91:1380-1382

22. Oxborough D, Heemels A, Somauroo J, McClean G, Mistry P, Lord R et al (2016) Left and right ventricular longitudinal strainvolume/area relationships in elite athletes. Int J Cardiovasc Img 32:1199-1211

23. Szauder I, Kovács A, Pavlik G (2015) Comparison of left ventricular mechanics in runner versus bodybuilders using speckle tracking echocardiography. Cardiovasc Ultrasound 13:7

24. MacIver DH, Townsend M (2008) A novel mechanism of heart failure with normal ejection fraction. Heart 94:446-449

25. Sengupta PP, Tajik AJ, Chandrasekaran K, Khandheria BK (2008) Twist mechanics of the left ventricle: principles and application. JACC Cardiovasc Imaging 1:366-376

26. Weiner RB, Weyman AE, Khan AM, Reingold JS, Chen-Tournoux AA, Scherrer-Crosbie M et al (2010) Preload dependency of left ventricular torsion: The impact of normal saline infusion. Circ Cardiovasc Imaging 3:672-678

27. Chang SA, Kim HK, Kim DH, Kim JC, Kim YJ, Kim HC et al (2010) Left ventricular twist mechanics in patients with apical hypertrophic cardiomyopathy: assessment with 2D speckle tracking echocardiography. Heart 96:49-55

28. Doucende G, Schuster I, Rupp T, Startun A, Dauzat M, Obert P et al (2010) Kinetics of left ventricular strains and torsion during incremental exercise in healthy subjects. Circ Cardiovasc Imaging 3:586-594 